\title{
Nonlinear cancellation in weakly coupled MEMS resonators
}

\author{
Milind Pandit, Chun Zhao, Aref Mustafazade, Guillermo Sobreviela and Ashwin A Seshia \\ Nanoscience Centre, Department of Engineering, University of Cambridge, \\ $11 \mathrm{JJ}$ Thomson Avenue, Cambridge CB3 OFF
}

\begin{abstract}
For the first time, this paper demonstrates the cancellation of nonlinear response in weakly coupled resonators. It has been observed that by working in the region where the mechanical nonlinearity of the resonators and the electrical nonlinearity of the electrostatic coupling cancel, the output current amplitudes are increased by $4 x$ while the trend of vibration amplitude variation upon stiffness perturbations is preserved as seen in the linear regime of the resonators.
\end{abstract}

\section{INTRODUCTION}

Weakly couple MEMS resonators (WCRs) have been employed in device applications for eletro mechanical signal processing [1] [2] and sensors [3][4] . In the context of sensing, a MEMS configuration consisting of weakly coupled resonators utilising the principle of vibration mode localization has been proposed. This technique has shown an improvement in sensitivity by orders of magnitude compared to the conventional single resonator devices using resonant frequency shifts as a readout [3]-[5]. The WCRs use amplitude ratio measurements instead of frequency shift measurements employed by the standard resonator systems to acheive common mode rejection capabilities that provide first-order response immunity to ambient temperature and pressure variations[6],[7].

In the context of sensing, WCRs have only been characterized in the linear regime at low vibration amplitudes so far. High vibration amplitudes allow for a higher output signal that in turn results in higher signal to noise ratio (SNR) in a system that is dominated by electrical noise of the readout electronics. However, at high vibration amplitudes, the nonlinearities of the system become considerable and higher order nonlinear effects, e.g. those that are often described by the Duffing equation, arise due to physical sources that are related to geometric and material properties, and from the electromechanical transduction scheme employed. These effects produce amplitude-frequency $(a-f)$ dependancy where multiple amplitudes and phase can be realized at a particular operating frequency. These nonlinearities limit the operation regime and power handling ability of the resonator system. Furthermore, they are undesirable in sensing applications due to their detrimental impact on the long-term stability of the resonators due to the upmixing of low frequency noise in the form of close-to-carrier noise [8].

In this work, using the concept of nonlinearity cancellations shown for a single resonator[9],[10], electrical and mechanical nonlinearity were compensated in WCRs by tuning the coupling voltage. When operating in this regime, a higher output signal is achieved without compromising on the sensitivity to stiffness perturbations as compared to a linear response. Since the current measurement setup is dominated by electrical noise of the readout electronics, this allows for a greater Signal to Noise Ratio (SNR) and a pathway to higher resolution amplitude ratio measurements.

\section{THEORY}

The WCRs can be schematically represented by a simple coupled spring-mass system as shown in Fig. 1. Such a system has two vibration modes - the in-phase mode and the antiphase mode. Assuming a weak coupling $\left(K_{c}<<K\right)$, the anti-phase mode is separated from the in-phase mode by a factor proportional to the coupling stiffness. Since the coupling is negative in this case, the anti-phase mode has a lower eigenfrequency than the in-phase mode as expected of such a system.

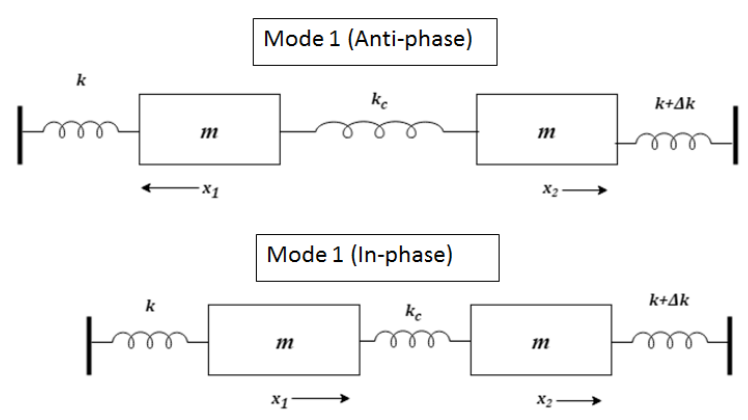

Fig. 1. Modes of Vibration for the coupled system.

The sources of mechanical nonlinearity in this system generally arise from the geometrical and material properties of the resonators themselves. Mechanical nonlinearities in the WCRs are similar to those that arise in single resonator systems and effect all of the modes of the device. These nonlinearities cause spring stiffening and result in positive $a-f$ effects. The nonlinear spring constant with mechanical nonlinearities for a DETF resonator can be approximated as [11]:

$$
\begin{gathered}
k(x)=k_{0}\left(1+k_{2} x^{2}\right) \\
k_{2}=\frac{0.767}{w^{2}}
\end{gathered}
$$

Since the operating mode of the system is the anti-phase mode, it is assumed that a complete cancellation of the second order mechanical nonlinear term occurs in a DETF resonator. 
Electrical nonlinearities on the other hand arise from the electrostatic force created by capacitive transduction, electrostatic forces and, coupling between the resonators. They cause spring softening and result in negative a-f effect. The nonlinearties arising due to capacitive transduction are similar to single resonator systems and affect all modes of the device. The nonlinear spring constant due to electrostatic forces can be derived as:

$$
k_{e n}\left(x_{i}\right)=-\frac{V_{D C}^{2}}{2} \frac{\partial^{2} C}{\partial x_{i}^{2}} \quad i=1,2
$$

The electrostatic forces also affect both the modes equally. However, the nonlinearites arising from the capacitive coupling effect the anti-phase mode more than the in-phase mode. In the in-phase mode, the two capacitve plates are moving inphase with each other and nonlinearities due to the coupling are suppresed. However, in the anti-phase mode, due to the capacitive plates moving out of phase with each other, the nonlinearities due to electrostatic coupling force arise and act as an additional contributor to the electrical nonlinearities of that mode. This nonlinear coupling spring constant can be derived as:

$$
k_{c n}=-\frac{V_{\text {couple }}^{2}}{2} \frac{\partial^{2} C}{\partial\left(x_{1}-x_{2}\right)^{2}}
$$

Due to the selective effects of the nonlinearity of coupler on the anti-phase mode, this mode is used for all experiments and simulation. Since the strength of the coupler can be tuned with the coupling voltage, the sources of electrical nonlinearities can be tuned to cancel the mechanical nonlinearities sources at a particular range of coupling voltages in mode 1 .

\section{Simulation}

A simulation was designed on Simulink ${ }^{\circledR}$ using the equations of motion of the nonlinear coupled resonator system driven from only one resonator:

$$
\begin{array}{r}
m \ddot{x}_{1}+c \dot{x}_{1}+k_{0} x_{1}+k_{2} x_{1}^{3}+k_{e n} x_{1}+k_{c n}\left(x_{1}-x_{2}\right)=F \cos (\omega t+\phi) \\
m \ddot{x}_{2}+c \dot{x}_{2}+k_{0} x_{2}+k_{2} x_{2}^{3}+k_{e n} x_{2}-k_{c n}\left(x_{1}-x_{2}\right)=0
\end{array}
$$

In Equation 5 and 6, it is assumed that the two resonators are identical thus resulting in the same linear and nonlinear mechanical stiffness terms. Furthermore, the coupling force is not split up into nonlinear terms but is used with a double differential term as shown in Equation 4 to preserve the effects of higher order nonlinear terms. The damping constant is chosen to have a quality factor of 10000 which, is similar to the experimentally observed quality factor. DC biasing voltage similar to that used in the experiment is applied to the resonators to simulate the experimental conditions.

A block diagram approach was used to represent the various forces being applied on the two resonators in terms of their displacements $x_{1}$ and $x_{2}$. The frequency response of resonator 1 at various coupling voltages driven at the same ac voltage of $125 \mathrm{mV}$ is shown in Fig. 2. A clear transition is seen with mechanical $a-f$ effect observed at $V_{\text {couple }}=12 \mathrm{~V}$ to electrical a-f effect observed at $V_{\text {couple }}=42 \mathrm{~V}$ with the cancelled response at $V_{\text {couple }}=27 \mathrm{~V}$.

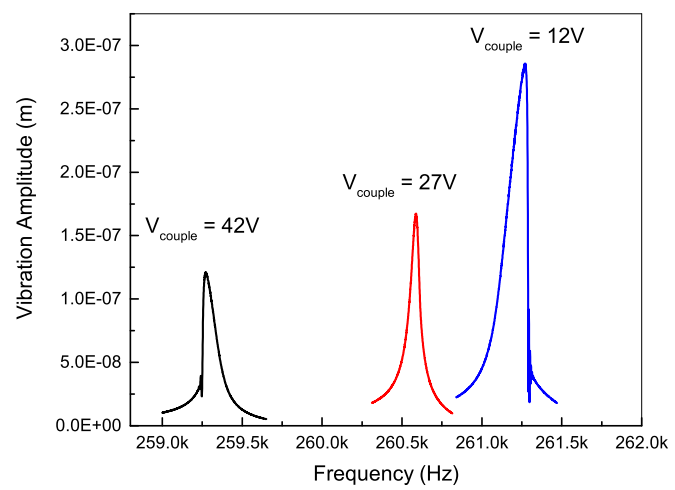

Fig. 2. Simulated frequency response curves of Mode 1 at $125 \mathrm{mV}$ AC drive voltage over three different coupling voltages showing mechanical nonlinearity, cancellation regime and electrical nonlinearity

Although this simulation identifies the cancellation phenomenon, it also includes the occurrence of mode localisation where the amplitude of vibration of the resonator changes (in this case decreases) by tuning the coupling voltage. This would mean that the amplitude of vibration $\left(x_{1}\right)$ at $V_{\text {couple }}=27 \mathrm{~V}$ is a lot lower than at $V_{\text {couple }}=12 \mathrm{~V}$ which leads to a lower nonlinear $a-f$ behavior. To truly understand the nonlinear cancellation behavior in the WCRs, an experimental study of the power handling ability is done which is discussed in the reults section.

\section{EXPERIMENT}

\section{A. Device Description}

The experiment is conducted using two identical, coupled double ended tuning fork (DETF) resonators. An optical micrograph of the device is shown in Fig. 3. The device was fabricated by MEMSCAP Inc. using SOIMUMPS, a commercial foundry process that uses silicon-on-insulator (SOI) wafers. The dimensions of the device are described in Table I.

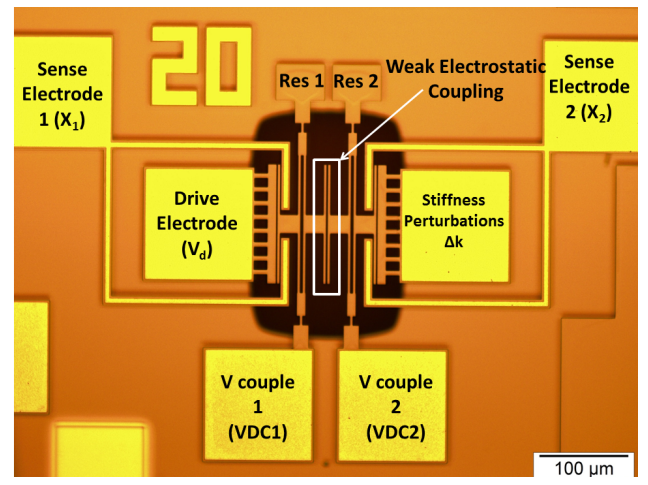

Fig. 3. Optical Micrograph of the device. 
TABLE I

Device Parameters

\begin{tabular}{lc}
\hline \multicolumn{1}{c}{ Parameter } & Dimensions \\
\hline & $350 \mu \mathrm{m}$ \\
Beam Length & $6 \mu \mathrm{m}$ \\
Beam Width & $260 \mu \mathrm{m}$ \\
Electrode Length & $6 \mu \mathrm{m}$ \\
Electrode Width & $25 \mu \mathrm{m}$ \\
Device Layer Thickness & $40 \mu \mathrm{m} \times 40 \mu \mathrm{m}$ \\
Proof Mass (2 for each DETF) & $2 \mu \mathrm{m}$ \\
Electrode Gaps & \\
\hline
\end{tabular}

\section{B. Measurement Setup}

The experimental setup is shown in Fig. 4. and measurements are conducted in vacuum. Two DC voltages (VDC1, VDC2) were applied on the two resonators to achieve a negative coupling stiffness, which was tuned by varying the potential difference. A network analyzer (Agilent 4396B) was used to apply an AC drive voltage $\left(V_{d}\right)$ along with a DC polarizing voltage on resonator 1 . The DC polarising voltage for drive and sense transductions are kept constant throughout the experiment. The output motional current of each resonator is passed through a transimpedance Amplifier (TIA), then through a Band Pass Filter (BPF) and finally fed back into the network analyzer to calculate the transmission $\left(S_{21}\right)$ parameter of the resonators. A negative stiffness perturbation relative to resonator 1 was applied on the perturbations electrode in the form of voltage differences for sensitivity measurements.

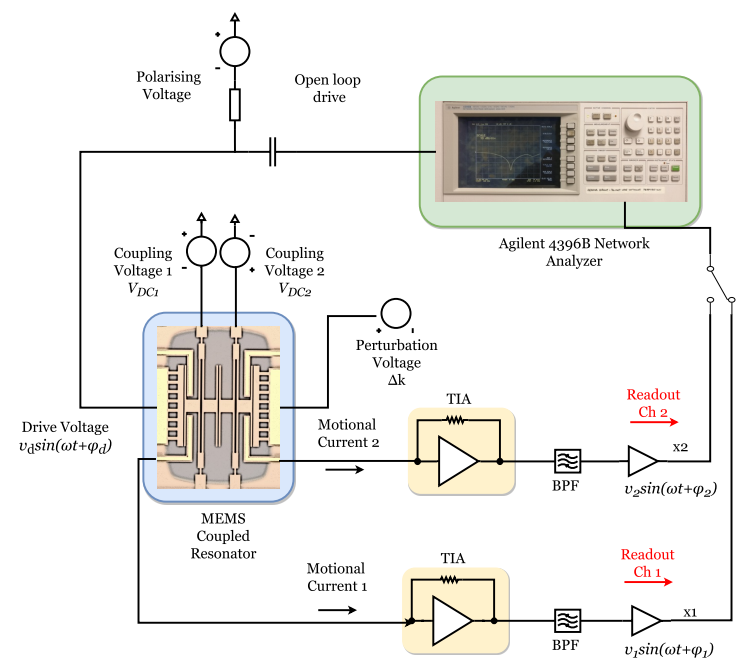

Fig. 4. Open loop measurmeent setup.

\section{RESUlts AND Discussion}

To understand the effect of the coupling voltage on the cancellation regime, the motional current for each coupling voltage is measured. In this experiment, the ac drive voltage is not kept constant but is varied to achieve the critical amplitude before bifurcation occurs. Experimentally, the bifurcation point of the system is located when the resonant frequency of the response shifts greater than half of the $3 \mathrm{~dB}$ bandwidth $\left(\Delta f_{\text {peak }} / \Delta f_{3 d B} \geq 0.5\right)$. From the point of bifurcation onwards, the $a-f$ effect becomes considerable such that the amplitude response is multi-valued and a hysteresis is described in the frequency response. This regime is not considered for this study.

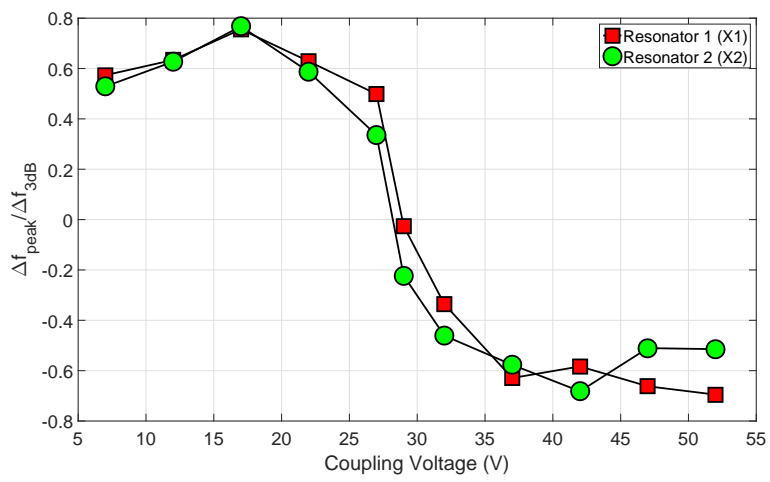

Fig. 5. Experimental ratio of peak frequency shift due to $a-f$ effect and $3 \mathrm{~dB}$ frequency (of the linear response at the same bias conditions) plotted over a swept coupling voltage in mode 1 .

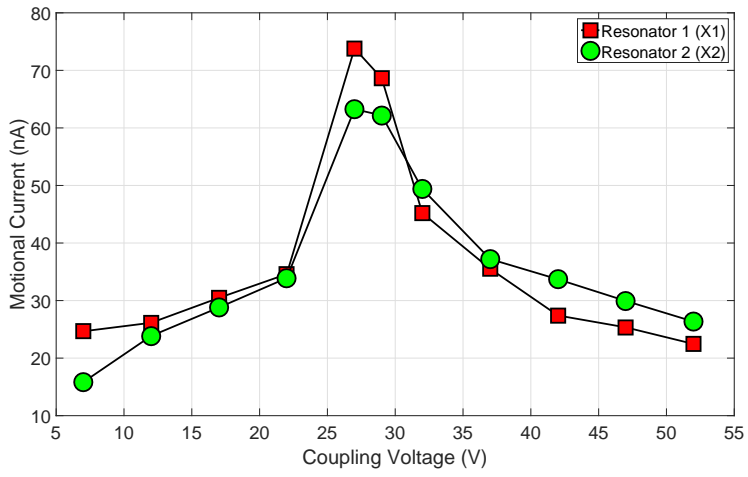

Fig. 6. Experimental motional current at critical displacement plotted against a swept coupling voltage showing non-linearity cancellation and enhancement in signal amplitude of mode 1. Peak motional current is observed between 25 and $35 \mathrm{~V}$ coupling voltage at $\mathrm{Vd}=125 \mathrm{mV}$.

The frequency response of the two resonator amplitudes $\left(X_{1}\right.$ and $\left.X_{2}\right)$ were measured in open loop configuration. The maximum motional current and $a-f$ effect at each coupling voltage were noted and plotted in Fig. 5 and Fig. 6. The a-f effect of mode 1 as seen in Fig. 5 reflects the cancellation occurring between $25 \mathrm{~V}$ and $35 \mathrm{~V}$. At lower coupling voltages, the mechanical nonlinearities dominate the response and a positive $a-f$ effect is seen. As the coupling voltage is increased, the electrical nonlinearities due to the coupler increase and compensate for the mechanical nonlinearites between $25 \mathrm{~V}$ and $35 \mathrm{~V}$. Upon further increasing the coupling voltage, the electrical nonlinearities start to dominate in the system and a negative a-f effect is observed. Similarly Fig. 6 describes the highest motional current achived at different coupling voltages before bifurcation occurs. Equivalent to Fig. 5, the peak motional current occurs between $25 \mathrm{~V}$ and $35 \mathrm{~V}$ where there is approximately $4 \mathrm{x}$ increase in the motional current (which translates to $12 \mathrm{~dB}$ improvement in SNR) compared to extreme coupling voltages of $7 \mathrm{~V}$ or $52 \mathrm{~V}$. Since the transduction voltages of drive and sense ports are kept constant throughout the experiment, the motional current is a good 
indicator of the trend of the motional response of the two resonators.

It is to be noted that the coupling stiffness plays a significant role in the sensitivity of the WCRs. The senstivity is inversely proportional to the coupling stiffness which signifies that it is beneficial to have a lower coupling voltages for sensing purposes. The resolution on the other hand is dependent on the amplitude of the output signal since the system is dominated by electrical noise from the amplifier circuit. Thus at lower coupling voltages, the system offers high sensitivity to stiffness perturbations but at the cancellation coupling voltage, the system offers higher output resolution. These two metrics are the most important specifications for sensor design and, in the case of WCRs, there is a tradeoff between the two metrics that needs to be considered before chosing the optimum coupling voltage for the application.

A final experiment was conducted to check the operation of the system as a sensor in the cancellation regime. The coupling voltage was set to $27 \mathrm{~V}$ and negative stiffness perturbations in terms of voltage difference were added to resonator 2 at the perturbation port. The voltages corresponding to the motional current of both resonators were recorded at each perturbation voltage for two drive voltage levels of $12.8 \mathrm{mV}$ (linear drive) and $125 \mathrm{mV}$ (nonlinear drive). The results are shown in Figure 7.

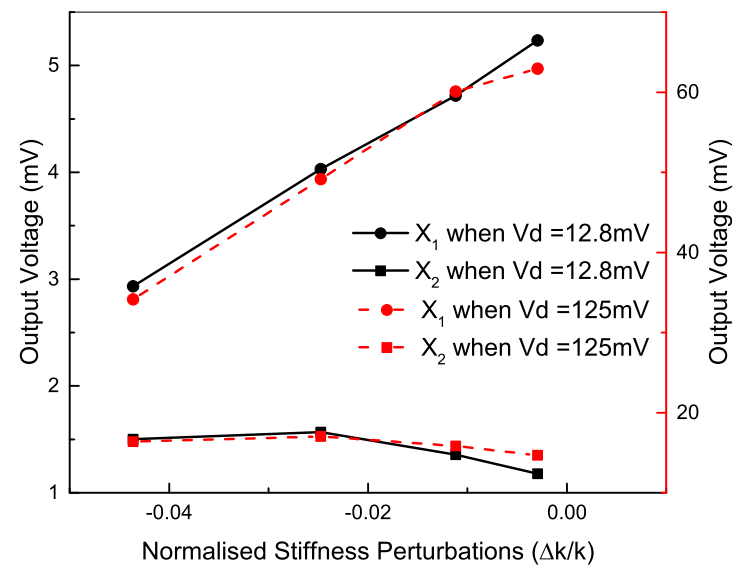

Fig. 7. Experimental amplitudes of vibration of the WCR in linear (12.8mV) and nonlinear AC drive $(125 \mathrm{mV})$ configurations. Output Voltage for nonlinear drive (in red and right axis) is higher than that of linear drive (in black and left axis) for both resonators X1 (circles) and X2 (squares)

The output voltage increases by a factor of $10 \mathrm{x}$ for both the resonators while conserving the trend of the change in amplitudes with respect to stiffness perturbations. When being used as a sensor, these stiffness perturbations can be a result of a force acting on one of the resonators due to change in tilt, acceleration or charge and thus, are a representative of any sensing applications these resonators will be used for. Since the output signal increased without compromisng the senstivity of the device, operating the system in the cancellation regime increases the signal to noise ratio and could be a potential gateway to higher resolution measurements.

\section{CONCLUSION AND FUTURE WORK}

In this work, we have demonstrated the concept of nonlinearity cancellation in WCRs through tuning the coupling voltage. The anti-phase mode is affected by the nonlinearities arising from the negative coupling while they are suppressed in the in-phase mode. It is seen that the motional current increases by a factor of $4 \mathrm{x}$ when the mechanical and electrical nonlinearities are compensated in the anti-phase mode. When being used as a sensor in the cancellation regime, the amplitude response for nonlinear drive is 10x higher than for a linear drive while conserving the trend of amplitude shift. This signifies a potential increase in the resolution of the amplitude measurements without compromising the sensitivity to stiffness perturbations.

Although open loop measurements allow for experimental characterization of the nonlinear cancellation phenomenon, a closed loop approach to tracking the resonant frequency is necessary for its application as a sensor or a timing reference. Parallel to this work, a closed loop approach for WCRs has been developed using a Phase Locked Loop approach and further characterizations are being made with respect to the optimum coupling for the best sensitivity and resolution.

\section{ACKNOWLEDGEMENTS}

This work is supported by funding from Innovate UK and Natural Environment Research Council.

\section{REFERENCES}

[1] S. Pourkamali and F Ayazi,'Electrically coupled MEMS bandpass filters: Part II. Without coupling element",Sens. Actuators Phys., vol. 122, pp. 317-325, Aug. 2005.

[2] D. K. Agrawal, J. Woodhouse, and A. A. Seshia. "Observation of locked phase dynamics and enhanced frequency stability in synchronized micromechanical oscillators." Phys. Rev. Lett., vol. 111, no. 8, p. 084101, Aug. 2013.

[3] H. Zhang, B. Li, W. Yuan, M. Kraft and H. Chang, "An Acceleration Sensing Method Based on the Mode Localization of Weakly Coupled Resonators," J. Microelectromechanical Syst., vol. 25. no. 2,pp. 286-296, Apr. 2016.

[4] P. Thiruvenkatanathan, J. Yan, and A. A. Seshia, "Enhancing Parametric Sensitivity in Electrically Coupled MEMS Resonators," J. Microelectromech. Syst., vol. 18, no. 5, pp. 10771086, Oct. 2009.

[5] C. Zhao, M. H. Montaseri, G. S. Wood, S. H. Pu, A. A. Seshia, and M. Kraft, "A Review on MEMS Coupled Resonators for Sensing Applications Utilizing Mode Localization," Sens. Actuators Phys., vol. 249, no. 5, pp. 93-111, Oct. 2016.

[6] P. Thiruvenkatanathan, J. Yan and A. A. Seshia, "Differential amplification of structural perturbations in weakly coupled MEMS resonators," IEEE Trans. Ultrason. Ferroelectr. Freq. Control, vol. 57. no. 3,pp. 690697, Mar. 2010.

[7] H. Zhang, J. Zhong, W. Yuan, J. Yang and H. Chang, "Ambient pressure drift rejection of mode-localized resonant sensors," in 2017 IEEE 30th International Conference on Micro Electro Mechanical Systems (MEMS), 2017, pp. 1095-1098.

[8] V. Kaajakari, J. K. Koskinen, and T. Mattila, "Phase noise in Capacitively Coupled Micromechanical Oscillators," IEEE Trans. Ultrason. Ferroelectr. Freq. Control, vol. 52. no. 12,pp. 2322-2331, Dec. 2005.

[9] M. Agarwal et al., "Non-linearity cancellation in MEMS resonators for improved power-handling," IEDM Technical Digest., 2005, pp. 286-289.

[10] M. Agarwal et al., "Nonlinear Characterization of Electrostatic MEMS Resonators," IEEE International Frequency Control Symposium and Exposition, 2006, pp. 209-212.

[11] V. Kaajakari, T. Mattila,A. Oja, and H. Seppä, "Nonlinear Limits for Single-Crystal Silicon Microresonators," J. Microelectromech. Syst., vol. 13. no. 5,pp. 715-724, Oct. 2004. 